\title{
Pädagogische Orientierungen zum Schriftspracherwerb von Fachkräften im Kindergarten (2006 und 2016)
}

\author{
Astrid Rank (iD
}

Online publiziert: 10. August 2020

(C) Der/die Autor(en) 2020

Zusammenfassung Die vorliegende Studie stellt eine Replikation des quantitativen Teils der Studie von Rank $(2008,2009)$ dar, in der die bei Fachkräften vorhandenen pädagogischen Orientierungen zum vorschulischen Lernen und Schriftspracherwerb untersucht wurden. An der erneuten Erhebung im Jahr 2016 mit demselben Fragebogen nahmen 147 Fachkräfte teil. Die Ergebnisse belegen eine nach wie vor hohe Zustimmung zum Kindergarten als Lernort. Selbstgesteuerter Schriftspracherwerb wird allerdings signifikant häufiger abgelehnt als noch vor zehn Jahren. Als signifikante Prädiktoren hierbei zeigen sich in der Regressionsanalyse ein instruierendes Lernkonzept und Unsicherheit gegenüber der Schule.

Schlüsselwörter Kindergarten · Pädagogische Fachkräfte · Schriftspracherwerb · Pädagogische Orientierungen

\section{Preschool teacher's beliefs about early literacy in kindergarten (2006-2016)}

\begin{abstract}
The present study is a replication of the quantitative part of Rank's study $(2008,2009)$, in which data about the pedagogical orientation of preschool teachers were collected. The replication reached 147 preschool teachers. The same questionnaire was used as in Rank 2008, 2009. There is still a high level of agreement with the kindergarten as a place of learning. The rejection of self-directed writing is significantly higher than ten years ago. The regression analysis shows an instructive learning concept and uncertainty towards school as significant predictors.
\end{abstract}

Keywords Kindergarten · Preschool teachers · Early literacy $\cdot$ Beliefs

Prof. Dr. A. Rank ( $\varangle)$

Lehrstuhl für Grundschulpädagogik, Institut für Bildungswissenschaft, Universität Regensburg, 93040 Regensburg, Deutschland

E-Mail: astrid.rank@ur.de 
In den Jahren 2005 und 2006 wurde eine Studie zu ,subjektiven Theorien von Erzieherinnen zu vorschulischem Lernen und Schriftspracherwerb“ durchgeführt (Rank 2008, 2009). Ein Ergebnis der Studie war, dass die Fachkräfte dem frühen Schriftspracherwerb im Kindergarten positiv gegenüber standen, v. a. der phonologischen Bewusstheit oder dem Schreiben des eigenen Namens. Es gab aber auch ablehnende Einstellungen v.a. gegenüber den schulnahen Bereichen wie dem phonetischen Schreiben oder dem frühen Lesen. Seitdem sind mehr als 10 Jahre vergangen, in denen sich Bildungspläne mit Betonung des Kindergartens als Lern- und Förderort etabliert haben. In einer Replikationsstudie wurde daher der quantitative Teil der Studie wiederholt, der sich auf die pädagogischen Orientierungen bezieht. Es wurde untersucht, ob sich die Ergebnisse aus Rank $(2008,2009)$ immer noch bestätigen.

\section{Vorschulisches Lernen und Schriftspracherwerb}

Vorwissen erleichtert den Aufbau neuen Wissens (vgl. u. a. Hasselhorn und Gold 2017). Allgemeinwissen ebenso wie bereichsspezifisches Wissen sind prädiktiv für die Schulleistungen. In den meisten Studien erweist sich das Vorwissen sogar als bedeutsamer als die allgemeine kognitive Grundfähigkeit (zusf. Stern und Grabner 2014; Fina 2017). Mit Blick auf den Schriftspracherwerb gelten vor allem die bereichsspezifischen Lernvoraussetzungen als ausschlaggebend. Im Bereich der Sprache werden diese in den „sprachlichen Basisqualifikationen“ (Ehlich et al. 2008, S. 18) zusammengefasst. Die Bedeutung der sprachlichen und schriftsprachlichen (Vorläufer-)fähigkeiten ist unbestritten. Einen hohen Stellenwert in der Forschung nimmt seit Jahrzehnten die phonologische Informationsverarbeitung ein, die sich als sehr spezifische und wichtige Vorläuferfähigkeit erwies (vgl. u. a. Schneider 2004). Aber auch die frühe Schriftkenntnis bzw. Wissen über und Erfahrungen mit Schrift (vgl. u.a. Ennemoser et al. 2012; Diamond et al. 2008; Lenel 2005), das Satzverständnis (vgl. Kotzerke et al. 2013) oder die Satzgedächtnisleistung (vgl. Goldammer et al. 2010) gelten als bedeutsam. Dabei wirken sich die jeweiligen Prädiktoren unterschiedlich und zu verschiedenen Zeiten aus. Während z. B. phonologische Bewusstheit, frühes Schriftwissen und Buchstabenkenntnis im frühen Schriftspracherwerb bedeutsam sind, gewinnen sprachliche Fähigkeiten wie der Wortschatz erst zum Ende der Grundschulzeit an Bedeutung (vgl. Ehri und Roberts 2006; Ennemoser et al. 2012). Diese Zusammenhänge sind durch die verschiedenen Kompetenzen erklärbar, die Schülerinnen und Schüler im Laufe des Schriftspracherwerbs benötigen und somit auch von der Unterrichtsmethode abhängig. Zum Dekodieren sowie zum alphabetischen Verschriften am Anfang der Schulzeit benötigt man andere Fähigkeiten (z.B. die phonologische Bewusstheit) als zum flüssigen Lesen und Rekodieren im weiteren Verlauf (z. B. syntaktische Kenntnisse). Insgesamt nimmt der Einfluss der vorschulischen Prädiktoren im Laufe der Grundschulzeit stark ab (vgl. Weinert und Stefanek 1997).

Unbestritten ist jedoch die Bedeutsamkeit der Vorläuferfähigkeiten insgesamt. Es ist auch evident, dass die Vorläuferfähigkeiten bei Schuleintritt sehr unterschiedlich ausgeprägt sind. Die Unterschiede in den individuellen Lernvoraussetzungen sind abhängig von Merkmalen der familiären Herkunft (zusammenf. in Neumann et al. 
2014; Liebers 2016; Kotzerke et al. 2013), und zwar sowohl direkt, etwa durch unterschiedliche Anregung und Unterstützung (z. B. Brühwiler und Helmke 2018), als auch indirekt, z. B. durch die Auswahl und Nutzung frühkindlicher Bildungseinrichtungen (vgl. u.a. Becker 2016, 2010). Diese soziale Ungleichheit kann durch qualitativ hochwertige Kindertagesbetreuung kompensiert werden (vgl. Fuchs-Rechlin und Bergmann 2014; Becker 2010; Rauschenbach 2009; Roßbach 2005). Möglicherweise werden die Unterschiede zwischen den Kindern in den Kindergärten aber auch noch größer (vgl. Kotzerke et al. 2013). Becker (2016, S. 175) zieht aus seinen Ergebnissen zu den Bildungseffekten vorschulischer Erziehung und Elementarbildung daher auch den Schluss: Vorschulische Einrichtungen ,,sind ein wirkungsvolles Instrumentarium unter mehreren Möglichkeiten, um Chancenungleichheiten zu reduzieren, und es dürfte - sollte die Verringerung von sozialer Ungleichheit von Bildungschancen ein bildungspolitisches Ziel darstellen - sinnvoll sein, dieses Programm quantitativ und qualitativ auszubauen“. Eine kompensatorische Wirkung der Kindertagesbetreuung ist allerdings nur bei hoher Qualität erreichbar (vgl. FuchsRechlin und Bergmann 2014).

Zusammenfassend lässt sich also feststellen, dass den kindlichen Vorläuferfähigkeiten eine große Bedeutung vor allem für den Schulerfolg der ersten Schuljahre zukommt. Hier sind z.B. Kompetenzen wie die frühe Schriftkenntnis zentral. Des Weiteren ist deutlich, dass der Kindergarten hier die auf sozialen Hintergründen beruhenden Ungleichheiten ausgleichen könnte. Eine Voraussetzung dafür ist allerdings, dass die dort vorzufindenden Angebote qualitativ hochwertig sind. Dies ist nicht nur aus der Forschung begründet, sondern auch vermittelt durch Bildungspläne politisch gewollt.

\section{Pädagogische Orientierungen von pädagogischen Fachkräften}

Die Umsetzung hochwertiger Angebote ist von den Kompetenzen der durchführenden Personen, also von den pädagogischen Fachkräften, abhängig. Es gibt ein Bündel an individuellen Kompetenzbereichen, die das pädagogische Handeln beeinflussen. Wissen, Einstellungen und Handlungskompetenzen sind hier zu nennen. Im Folgenden werden die pädagogischen Orientierungen in den Blick genommen, die, so vermuten u. a. Mischo et al. (2012) mit Blick auf empirische Befunde, sich direkt auf die Prozessqualität und so vermittelt auf die kindliche Entwicklung auswirken können.

Pädagogische Orientierungen sind nach Tietze (1998, S. 22) die ,pädagogischen Vorstellungen, Werte und Überzeugungen der an den pädagogischen Prozessen unmittelbar beteiligten Erwachsenen“. Bedeutsam ist die Orientierung der pädagogischen Fachkraft, da das „Bild vom Kind“, das die Fachkraft vertritt ,in ihrer Auffassung über Bildung und Entwicklung sowie im konkreten Erziehungsziel sowie Erziehungsmaßnahmen zum Ausdruck kommt" (Becker-Stoll und Wertfein 2013, S. 808). Eine Orientierung oder Überzeugung, verstanden als teilweise implizite und unbewusste, relativ stabile und möglicherweise widersprüchliche kognitive Struktur, die im Sinne von Theoriebildung Situationen definiert, Ereignisse nachträglich erklärt, künftige Ereignisse prognostiziert und Handlungen daraus ableitet, kann man 
auch als „subjektive Theorie“ bezeichnen. Eine „subjektive Theorie“ subsummiert also pädagogische Vorstellungen über das Lernen und die Entwicklung von Kindern sowie epistemologische Vorstellungen. Hinzu kommt die Haltung zu den Lerngegenständen (hier dem Schriftspracherwerb). Zur subjektiven Theorie werden diese Überzeugungen, weil sie im Sinne von Theoriebildung herangezogen werden, um bestimmte Ereignisse und Tatsachen zu erklären, Regeln und Gesetzmäßigkeiten abzuleiten und Handlungen zu begründen. Im Unterschied zu wissenschaftlichen Theorien sind allerdings die der Theoriebildung zugrunde liegenden „Daten“ persönlicher Erfahrung und Interpretation entnommen. Sie sind individuell und werden nicht unbedingt zwischen Subjekten geteilt.

Im Folgenden werden die Ergebnisse verschiedener Studien zu subjektiven Theorien von pädagogischen Fachkräften, aber auch zu benachbarten Konstrukten kurz dargestellt, bevor ausführlicher die zu replizierende eigene Studie vorgestellt wird (Rank 2008).

Unter dem Begriff „Subjektive Theorien von pädagogischen Fachkräften im Kindergarten“ wurden in den letzten Jahren nur einzelne Studien durchgeführt. Hierbei handelt es sich in der Regel um kleinere qualitative Studien, die im Rahmen von Dissertationen angefertigt wurden (z. B. Brandl 2016; von Bülow 2011). In diesen beiden Studien wird die Schulvorbereitung als Ziel der Kindergartenarbeit genannt. Beide bescheinigen aber auch die hohe Ambivalenz der Fachkräfte: Lehrgänge werden eher abgelehnt, der Kindergarten soll die Kulturtechniken nicht vermitteln (vgl. von Bülow 2011). Die jüngere Studie von Brandl geht noch weiter und berichtet (trotz der Bildungspläne, die Anschlussfähigkeit garantieren sollen) von einer hohen Unsicherheit und einer daraus resultierenden Abwehrhaltung gegenüber dem schulischen Lernen (vgl. Brandl 2016). Vorschulische Bildungsprogramme würden nicht an den Interessen der Kinder anknüpfen, ,der dadurch forcierte kognitive Fähigkeitserwerb steht in den Wahrnehmungen einem für den Kindergarten typischen individuellen, sozial-emotionalen Lernen entgegen“ (Brandl 2016, S. 273).

Ergebnisse weiterer Studien, die die Überzeugungen von Erzieher*innen in den Blick nehmen, hier v.a. die BIKS-Studie (vgl. Faust 2013), runden dieses Bild ab. Die Förderung im Kindergarten wird von den befragten Fachkräften als ,ganzheitlich“ beschrieben. Persönlichkeits- und Kreativitätsentwicklung sind am wichtigsten. Schulvorbereitung gilt eher als unwichtig. Diese wird lediglich im letzten Kindergartenjahr für die Vorschulkinder als gesondertes, abgetrenntes Angebot bereitgestellt. Je näher die Ziele dem Kompetenzerwerb in der Schule sind und Lehrgangs- oder instruierenden Charakter haben (z. B. Schriftvermittlung), desto mehr werden sie in Frage gestellt (zusammenf. Wehner und Kratzmann 2013). Nach den Kriterien für Schulfähigkeit befragt, nennen die Fachkräfte, Lehrpersonen und Eltern Aspekte wie Ausdauer und Konzentrationsfähigkeit, den kognitiven Entwicklungsstand und Selbstständigkeit. Proximale Lernvoraussetzungen, z. B. auch die Kenntnis von Buchstaben und Zahlen, werden von den Fachkräften im Kindergarten nicht erwähnt. Zwar führen die Autorinnen diesen Befund auch auf die Fragestellung in der Studie bzw. die gegebenen Antwortmöglichkeiten zurück. Dennoch ist festzustellen, dass die enorme Bedeutsamkeit der proximalen Vorläuferfähigkeiten und die Wichtigkeit ihrer vorschulischen Förderung nicht bekannt zu sein scheinen (vgl. PohlmannRother et al. 2013). Plehn (2013, S. 228) sieht in den Überlegungen der pädagogi- 
schen Fachkräfte zur Einschulung ,,normative Konzepte von Kindheit“, die v. a. freie Entfaltung, freies Spiel als Indikatoren einer unbeschwerten Kindheit enthalten.

International orientiert sich die Forschung in erster Linie am Konstrukt der „Beliefs“ (am ehesten übersetzbar mit ,,pädagogische Überzeugungen“). Unter der Prämisse, dass Beliefs handlungsleitend sind (zusammenf. in Hamre et al. 2012), werden sowohl Beliefs erhoben als auch ihre Veränderung erforscht. Terminologisch werden Einrichtungen für Kinder zwischen 3 und 6 Jahren in nicht-deutschen Bildungssystemen häufig als „Schule“ oder „Vorschule“ bezeichnet (z. B. „École Maternelle“, „Förskola“, „Nursery School“). Damit liegt die Vermutung nahe, dass die Vorschule als erste Stufe des Bildungssystems verstanden wird und entsprechend keine große Ablehnung einer eher lernzielorientierten Vorgehensweise im Kindergarten zu finden ist. Doch auch international ist v.a. in Studien aus westlichen Ländern eine Ablehnung von Schulvorbereitung im Sinne von Schriftvorbereitung zu finden (z.B. in Kanada: Lynch und Owston 2015) sowie eine hohe Zustimmung zu kindorientiertem, nicht instruierendem Vorgehen, wobei die Zustimmung zu instruierendem Vorgehen sehr stark variiert (z. B. in den USA: Wen et al. 2011). Wang et al. (2008) berichten in einer Studie mit 296 chinesischen und 146 US-amerikanischen Vorschullehrkräften von einer großen Ähnlichkeit in den Beliefs der untersuchten Personen. Dass die Lerninitiative vom Kind ausgehen soll, findet hohe Zustimmung. Der Bedeutsamkeit von schulnahen Inhalten wird beigepflichtet. Allerdings stimmen die amerikanischen Fachkräfte tendenziell eher einem informellen, weniger strukturierten Vorgehen zu, während die chinesischen Fachkräfte ein eher vermittelndes, an schulnahen Inhalten angelehntes Vorgehen bevorzugen. Dies zeigt sich insbesondere beim Lernen der Schrift, was aber auch im wesentlich komplexeren chinesischen Schriftsystem begründet sein kann. Abry et al. (2015) untersuchten in den USA, ob Vorschul- und Grundschullehrkräfte ähnliche Beliefs über die Bedeutsamkeit schulrelevanter Fähigkeiten hatten. Es zeigte sich, dass die Übereinstimmung in den akademischen Kompetenzen am meisten differierte und sich die mangelnde Übereinstimmung tatsächlich auch negativ auf die kognitive und soziale Entwicklung der Kinder auswirkte, v. a. auf diejenigen mit schwächeren Lernvoraussetzungen.

Man kann also zusammenfassen: Auch aktuelle Studien zu pädagogischen Orientierungen zeigen, dass frühpädagogische Fachkräfte den Kindergarten als ganzheitlich wahrnehmen und eine direkte gezielte Förderung oder Instruktion, auch in den schulnahen Bereichen und insbesondere in Programmen, ablehnen. Unter Berücksichtigung der Bedeutung von Vorläuferfähigkeiten wie der phonologischen Bewusstheit und der frühen Schriftkenntnis, der Wirksamkeit qualitativ hochwertiger Angebote, die einer Planung und Vorbereitung bedürfen, und der nachgewiesenen Erfolge von einzelnen Trainingsprogrammen muss dies als eher ungünstig eingeschätzt werden. Wehner und Kratzmann (2013, S. 94) äußern sich dahingehend besorgt: „Da eine gezielte Förderung und schulvorbereitende Maßnahmen teilweise explizit abgelehnt werden, lässt sich vermuten, dass die Diskussion um die Bedeutung früher Fördermaßnahmen bisher nicht vollständig bei den Beteiligten , angekommen" ist".

2006, als die Bildungspläne gerade eingeführt wurden, zeigte sich bei einzelnen Fachkräften diesbezüglich eine deutlich positivere Haltung. Rank (2008) schildert in der Studie ,Subjektive Theorien von Erzieherinnen zu vorschulischem Lernen 
und Schriftspracherwerb", wie die Erzieherinnen den bayerischen Bildungs- und Erziehungsplan (BEP, Bayerisches Staatsministerium für Arbeit und Sozialordnung, Familie und Frauen (2006)) als erwünschte Legitimation nennen, um endlich vom von ihnen selbst so genannten „Basteltanten-Image“ wegzukommen (S. 276) und den Kindergarten zu einem Lernort aufzuwerten. Die damals neuen Inhalte und Begriffe wurden zwar nicht immer vollumfänglich verstanden, sondern tauchten mitunter auch als Worthülsen auf (z. B. „lernmethodische Kompetenz“). Aber der Kindergarten als Lernort wurde für die Fachkräfte transparent: „Unbedingt, unbedingt. In der öffentlichen Wahrnehmung und auch in unserer eigenen. Dass man sich besser eigentlich in den Vordergrund bringen muss. Sagen muss, natürlich lernen wir was und wie viel wir lernen!“ (Ankerbeispiel aus Rank 2008, S. 276). Allerdings war die Zustimmung auch nicht durchgängig gegeben. In einer Clusteranalyse zeigten sich drei verschiedene Erzieherinnentypen. Diese drei Typen unterschieden sich in ihrer Einstellung zum kognitiven Lernen ${ }^{1}$ und Schriftspracherwerb im Kindergarten, in ihrer Zusammenarbeit und Einstellung zur Schule und in ihrer Unsicherheit bezüglich der eigenen Diagnose- und Förderkompetenz im Schriftspracherwerb signifikant. Das Cluster 1 mit der Kennzeichnung „unsicher/schulnah/leicht lernskeptisch“ beinhaltete 39 Erzieherinnen und damit 26,71\% der für die Berechnung herangezogenen Fälle. Im Cluster 2 ,sicher/schulnah/lernorientiert“" waren 64 Erzieherinnen eingeordnet, was 43,84\% entsprach. Dem Cluster 3 ,sicher/schulfern/lernorientiert“ waren 43 Erzieherinnen zuzuordnen, das waren 29,45\% der Befragten. Vor allem das erste Cluster war hierbei bedenklich. Denn die Skepsis dieser Fachkräfte war am größten im Bereich des vorschulischen Schriftspracherwerbs. Sie arbeiteten gut mit der Schule zusammen, waren aber in ihrer eigenen sprach- und schriftsprachlichen Diagnose- und Förderkompetenz unsicher. In einem Fallbeispiel konnte gezeigt werden, dass bei einer Erzieherin dieses Clusters die Tendenz bestand, der Schule nichts vorwegnehmen zu wollen. Kinder, die bereits Interesse an Schulinhalten, wie etwa dem Schriftspracherwerb, zeigten, wurden nicht positiv unterstützt.

Insgesamt ergaben Regressionsanalysen, dass eine entsprechende Ablehnung kognitiven Lernens im Kindergarten mit einer negativen Einstellung zum Schriftspracherwerb im Kindergarten und einem instruierenden Lernkonzept korrelierte. Die Bearbeitung eines Fallbeispiels eines phonetisch schreibenden Kindergartenkindes ergab, dass ein eher ablehnender, skeptischer Umgang mit dem phonetischen Schreiben signifikant mit Unsicherheit in der schriftsprachlichen Diagnose- und Förderkompetenz, einer negativen Einstellung zum kognitiven Lernen im Kindergarten und einem instruierenden Lernkonzept zusammenhing.

Seitdem sind mehr als 10 Jahre vergangen, in denen sich Bildungspläne etabliert haben, die den Kindergartens als Lern- und Förderort betonen. In einer Replikationsstudie wurde daher untersucht, ob sich die Ergebnisse aus Rank (2008, 2009) immer noch bestätigen würden. Deshalb wurden im Winter 2016 die Kindergärten aus der Stichprobe der ursprünglichen Studie erneut kontaktiert. Dabei wurden insgesamt 147 Fachkräfte erreicht, von denen 19 der ersten Studie zugeordnet werden konnten. Es wurde derselbe Fragebogen wie im Oktober 2005 und Juni 2006 ein-

\footnotetext{
${ }^{1}$ Kognitives Lernen ist hier und im Folgenden verstanden als Erwerb kognitiver Kompetenzen, etwa Begriffsbildung und Wissenserwerb.
} 
gesetzt. Da lediglich die quantitative Befragung wiederholt wurde, sich subjektive Theorien aber erst im qualitativ-empirischen Forschungsprozess erschließen, wird für die Ergebnisse der Replikationsstudie am Begriff der pädagogischen Orientierungen festgehalten.

\section{Eigene Forschung}

\subsection{Forschungsfragen und Design}

Im Artikel werden zwei Forschungsfragen verfolgt:

- Unterscheiden sich die pädagogischen Orientierungen zum Schriftspracherwerb der pädagogischen Fachkräfte von 2016 von denen von 2006?

- Beeinflussen die unabhängigen Variablen (Berufsjahre, Lernkonzept, Unsicherheit und Zusammenarbeit mit der Schule) die abhängigen Variablen „Einstellung zum selbstgesteuerten Schriftspracherwerb“ und „Umgang mit einem Kind, das phonetisch schreibt"“?

Zur Beantwortung der ersten Frage wurde mit den Daten der Personen, die an beiden Studien teilgenommen hatten, wurde eine Varianzanalyse mit Messwiederholung gerechnet.

Die zweite Fragestellung ergibt sich aufgrund der Ergebnisse von Rank (2008). Dort konnte die Ablehnung des selbstgesteuerten Schriftspracherwerbs und der negative Umgang mit einem phonetisch schreibendem Kind über diese Variablen (Unsicherheit, Lernkonzept, Schulnähe) erklärt werden. Es wurde eine multivariate lineare Regressionsanalyse über die Daten des dritten Messzeitpunktes (2016) gerechnet. Im Artikel werden die Beziehungen zwischen den abhängigen Variablen „Einstellung zum selbstgesteuerten Schriftspracherwerb“ sowie „Umgang mit einem Kind, das im Kindergarten phonetisch schreibt“" und den unabhängigen Variablen wie „Berufsjahre“, „Lernkonzept“, „Unsicherheit“ und „Zusammenarbeit mit der Schule“ dargestellt. Zur weiteren Untersuchung der Zusammenhänge innerhalb der pädagogischen Orientierungen wurden die qualitativen offenen Fragen aus dem Fragebogen mit paraphrasierender Inhaltsanalyse ausgewertet und die Kategorien gruppiert.

\subsection{Instrumente}

Es wurde derselbe Fragebogen verwendet, der bereits 2005 (MZP1) und 2006 (MZP2) eingesetzt wurde. Der Fragebogen enthielt Skalen, deren Items auf einer vierstufigen Likertskala (,stimmt gar nicht“, „stimmt wenig“, „stimmt ziemlich“, „stimmt völlig“) anzukreuzen waren. Tab. 1 zeigt die im Artikel verwendeten Skalen inklusive der Reliabilitäten und Skalenkennwerte an den drei Messzeitpunkten. 
Zusätzlich zu den in Tab. 1 vorgestellten Skalen konnten die teilnehmenden Erzieher*innen eine offene Frage beantworten ${ }^{2}$. Die Antworten $(n=74)$ wurden von zwei Kodiererinnen offen induktiv in der Technik der paraphrasierenden Inhaltsanalyse kodiert. Während die erste Kodiererin zunächst die induktiv entstandenen Kategorien festlegte, kodierte die zweite Kodiererin dasselbe Material mit den zuvor festgelegten Kategorien. Als Kodiereinheit galten Sätze und Satzteile, insgesamt gab es 113 kodierte Einheiten. Die Interkoderreliabilität liegt bei einer prozentualen Übereinstimmung der Segmente bei $75 \%$.

\subsection{Stichprobe}

An der Studie in den Jahren 2005 und 2006 nahmen 195 pädagogische Fachkräfte aus 55 Kindergärten dreier bayerischer Landkreise teil. Im Jahr 2016 wurden erneut alle Kindergärten der Region zunächst telefonisch und dann per Email angefragt. Insgesamt sandten 147 pädagogische Fachkräfte den Fragebogen zurück. 19 Personen davon nahmen bereits an der ersten Studie teil. Somit liegen von $n=19$ Fragebogendaten zu drei Messzeitpunkten vor. Die anderen Fachkräfte $(n=128)$ nahmen zum ersten Mal an der Studie teil. Tab. 2 zeigt einen Überblick über die Stichprobe.

\subsection{Ergebnisse}

Es zeigen sich folgende Ergebnisse:

Frage 1 Unterscheiden sich die pädagogischen Orientierungen der pädagogischen Fachkräfte von 2016 von denen von 2006?

In Tab. 1 sieht man alle Mittelwerte der gesamten Stichproben über die drei Messzeitpunkte. Anschließend wurde eine Varianzanalyse mit Messwiederholung mit den Daten der 19 Fachkräfte erstellt, die an allen drei Messzeitpunkten teilgenommen hatten. Die Entwicklung entspricht derjenigen, die auch in den Mittelwerten aller Teilnehmenden (sichtbar in Tab. 1) aufscheint. So äußern die Fachkräfte eine hohe Zustimmung zum Kindergarten als Lernort und zu einem konstruktivistischen Lernkonzept. Die Einstellung zur Zusammenarbeit mit der Schule bleibt ebenfalls bestehen. Einerseits arbeiten die pädagogischen Fachkräfte gut mit den Lehrkräften zusammen, andererseits sind sie der Schule gegenüber tendenziell unsicher. Weiterhin wurden Skalen zur phonologischen Bewusstheit erhoben (Zuordnung der phonologischen Bewusstheit im engeren und weiteren Sinn, positive Bedeutung). Eine der Skalen wird exemplarisch in Tab. 1 dargestellt. Bereits 2005 und 2006 erfolgte die Zuordnung, v. a. der Bereiche der phonologischen Bewusstheit im weiteren Sinn, sehr sicher und die Zustimmung zu ihrer Bedeutung war hoch. Daran hat sich nichts verändert, so dass diese Skalen nicht weiter ausgewertet werden.

\footnotetext{
2 Hat sich durch Ihre Arbeit etwas

- an Ihrer Einstellung zum Lernen von Kindern,

- an Ihrer Einstellung zum Lesen- und Schreibenlernen von Kindern

- an Ihren Vorstellungen von phonologischer Bewusstheit

grundsätzlich verändert? Wenn ja, skizzieren Sie bitte kurz diese Veränderung und was dazu geführt hat.
} 
Tab. 1 Deskriptive Fragebogendaten (MZP1 2005, MZP2 2006, MZP3 2016)

\begin{tabular}{|c|c|c|c|c|c|}
\hline & $\begin{array}{l}\text { Anzahl } \\
\text { Items }\end{array}$ & Beispielitem & $\begin{array}{l}\text { Cronbachs } \\
\text { Alpha }\end{array}$ & $N$ & $\mathrm{MW}(\mathrm{SD})$ \\
\hline & & & MZP1 & MZP1 & MZP1 \\
\hline & & & MZP2 & MZP2 & MZP2 \\
\hline & & & MZP3 & MZP3 & MZP3 \\
\hline \multicolumn{6}{|c|}{ Skalen zum Lernkonzept } \\
\hline \multirow{3}{*}{$\begin{array}{l}\text { Positive Einstellung } \\
\text { zum kognitiven } \\
\text { Lernen im } \\
\text { Kindergarten }\end{array}$} & \multirow[t]{3}{*}{4} & \multirow{3}{*}{$\begin{array}{l}\text { Im Kindergarten gehört es } \\
\text { dazu, dass sich Kinder mit } \\
\text { Lerninhalten beschäftigen }\end{array}$} & 0,76 & 191 & $3,62(0,54)$ \\
\hline & & & 0,79 & 172 & $3,64(0,53)$ \\
\hline & & & 0,67 & 145 & $3,52(0,55)$ \\
\hline \multirow{3}{*}{$\begin{array}{l}\text { Instruierendes } \\
\text { Lernkonzept }\end{array}$} & \multirow[t]{3}{*}{3} & \multirow{3}{*}{$\begin{array}{l}\text { Durch Erklärungen lernt } \\
\text { ein Kind am meisten }\end{array}$} & 0,70 & 192 & $2,55(0,68)$ \\
\hline & & & 0,75 & 165 & $2,42(0,70)$ \\
\hline & & & 0,72 & 143 & $2,57(0,73)$ \\
\hline \multirow{3}{*}{$\begin{array}{l}\text { Konstruktivistisches } \\
\text { Lernkonzept }\end{array}$} & \multirow[t]{3}{*}{4} & \multirow{3}{*}{$\begin{array}{l}\text { Man sollte den Kindern } \\
\text { viel Zeit einräumen, eigene } \\
\text { Deutungen für Phänomene } \\
\text { zu suchen, auch wenn } \\
\text { dadurch falsche } \\
\text { Vorstellungen entstehen }\end{array}$} & 0,53 & 185 & $3,07(0,48)$ \\
\hline & & & 0,58 & 167 & $3,21(0,42)$ \\
\hline & & & 0,66 & 142 & $3,19(0,51)$ \\
\hline \multicolumn{6}{|c|}{ Skalen zum Schriftspracherwerb } \\
\hline \multirow{3}{*}{$\begin{array}{l}\text { Ablehnender Um- } \\
\text { gang mit Kindern, } \\
\text { die phonetisch } \\
\text { schreiben }\end{array}$} & \multirow[t]{3}{*}{7} & \multirow{3}{*}{$\begin{array}{l}\text { Ich halte das Kind davon } \\
\text { ab, noch mehr zu schreiben }\end{array}$} & 0,87 & 186 & $1,75(0,71)$ \\
\hline & & & 0,87 & 174 & $1,59(0,65)$ \\
\hline & & & 0,87 & 132 & $1,76(0,75)$ \\
\hline \multirow{3}{*}{$\begin{array}{l}\text { Ablehnung des } \\
\text { selbstgesteuerten } \\
\text { Schriftspracher- } \\
\text { werbs }\end{array}$} & \multirow[t]{3}{*}{6} & \multirow{3}{*}{$\begin{array}{l}\text { Wenn Kinder beim Lesen- } \\
\text { und Schreibenlernen } \\
\text { eigene Vorstellungen } \\
\text { entwickeln, wird der } \\
\text { Erwerb richtiger Muster } \\
\text { erschwert }\end{array}$} & 0,56 & 181 & $2,09(0,56)$ \\
\hline & & & 0,64 & 162 & $2,03(0,64)$ \\
\hline & & & 0,87 & 136 & $2,20(0,64)$ \\
\hline \multirow{3}{*}{$\begin{array}{l}\text { Unsicherheit bezüg- } \\
\text { lich Sprach- und } \\
\text { Schriftsprachent- } \\
\text { wicklung von } \\
\text { Kindergartenkindern }\end{array}$} & \multirow[t]{3}{*}{5} & \multirow{3}{*}{$\begin{array}{l}\text { Ich weiß nicht genau, wie } \\
\text { sich die Schriftsprache } \\
\text { beim Kind entwickelt }\end{array}$} & 0,73 & 187 & $2,21(0,59)$ \\
\hline & & & 0,79 & 171 & $1,97(0,56)$ \\
\hline & & & 0,75 & 144 & $1,88(0,57)$ \\
\hline \multicolumn{6}{|c|}{ Skalen zur phonologischen Bewusstheit (Beispiel) } \\
\hline \multirow{3}{*}{$\begin{array}{l}\text { Auswirkung der } \\
\text { phonologischen } \\
\text { Bewusstheit auf den } \\
\text { Schriftspracherwerb }\end{array}$} & \multirow[t]{3}{*}{4} & \multirow{3}{*}{$\begin{array}{l}\text { Wenn Kindergartenkinder } \\
\text { einzelne Laute aus Wörtern } \\
\text { heraushören können, } \\
\text { lernen sie in der Schule } \\
\text { leichter lesen und schreiben }\end{array}$} & 0,88 & 192 & $3,51(0,53)$ \\
\hline & & & 0,90 & 173 & $3,58(0,53)$ \\
\hline & & & 0,86 & 145 & $3,34(0,47)$ \\
\hline
\end{tabular}


Tab. 1 (Fortsetzung)

\begin{tabular}{|c|c|c|c|c|c|}
\hline & $\begin{array}{l}\text { Anzahl } \\
\text { Items }\end{array}$ & Beispielitem & $\begin{array}{l}\text { Cronbachs } \\
\text { Alpha }\end{array}$ & $N$ & MW (SD) \\
\hline & & & MZP1 & MZP1 & MZP1 \\
\hline & & & MZP2 & MZP2 & MZP2 \\
\hline & & & MZP3 & MZP3 & MZP3 \\
\hline \multicolumn{6}{|c|}{ Einfluss der Grundschule $^{a}$} \\
\hline \multirow{3}{*}{$\begin{array}{l}\text { Gute } \\
\text { Zusammenarbeit mit } \\
\text { der Schule }\end{array}$} & \multirow[t]{3}{*}{2} & \multirow{3}{*}{$\begin{array}{l}\text { Die Grundschullehrerinnen } \\
\text { und -lehrer, mit denen ich } \\
\text { zusammenarbeite, nehmen } \\
\text { meine Arbeit ernst }\end{array}$} & - & - & - \\
\hline & & & 0,88 & 166 & $3,05(0,82)$ \\
\hline & & & 0,85 & 145 & $3,13(0,78)$ \\
\hline \multirow{3}{*}{$\begin{array}{l}\text { Unsicherheit darüber, } \\
\text { wie Förderung im } \\
\text { Kindergarten durch } \\
\text { die Grundschulleh- } \\
\text { rerinnen gesehen } \\
\text { wird }\end{array}$} & \multirow[t]{3}{*}{5} & \multirow{3}{*}{$\begin{array}{l}\text { Ich habe Angst davor, } \\
\text { durch zu viel Förderung im } \\
\text { Kindergarten Inhalte der } \\
\text { Schule vorwegzunehmen }\end{array}$} & - & - & - \\
\hline & & & 0,64 & 162 & $2,00(0,60)$ \\
\hline & & & 0,40 & 138 & $1,98(0,52)$ \\
\hline
\end{tabular}

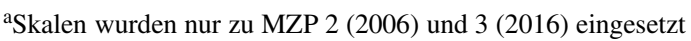

Eine signifikante Veränderung zwischen 2006 und 2016 zeigt sich jedoch in der Skala Ablehnung des selbstgesteuerten Schriftspracherwerbs. Trotz des konstruktivistischen Lernkonzepts und eines eher unterstützenden Umgangs mit Kindern, die phonetisch schreiben, halten die Fachkräfte selbstgesteuerten Schriftspracherwerb im Vorschulalter für weniger positiv als noch vor zehn Jahren. Tab. 3 zeigt die Werte dieser Skala für die 15 Teilnehmenden, von denen Werte aller drei Messzeitpunkte vorliegen.

Frage 2 Beeinflussen die unabhängigen Variablen (Berufsjahre, Lernkonzept, Unsicherheit und Zusammenarbeit mit der Schule) die abhängigen Variablen „Einstellung zum selbstgesteuerten Schriftspracherwerb“ und „Umgang mit einem Kind, das phonetisch schreibt"?

Hintergrund der multivariaten Regressionsanalyse war die Annahme, dass sich die pädagogischen Orientierungen von Fachkräften, die selbstgesteuerten Schriftspracherwerb im Kindergarten ablehnen, von den pädagogischen Orientierungen der anderen Fachkräfte unterscheiden. Die Zusammensetzung dieser Orientierungen lässt sich über den Einbezug verschiedener Skalen in eine Regressionsanalyse statistisch zeigen. Hierfür wurden lediglich die Fragebögen des dritten Messzeitpunktes, also von 2016, einbezogen. Getestet wurden als abhängige Variablen die ablehnende Einstellung zum selbstgesteuerten Schriftspracherwerb und der ablehnende Umgang mit einem Kind, das im Kindergarten phonetisch schreibt. Als unabhängige Variablen gingen die Jahre der Berufstätigkeit, die Zusammenarbeit mit der Schule, die Unsicherheit gegenüber der Schule, die Unsicherheit bezüglich der Sprachund Schriftsprachentwicklung von Kindern, ein instruierendes Lernkonzept und ein konstruktivistisches Lernkonzept ein. Die Voraussetzungsanalyse für die Regression ergab keine Auffälligkeiten.

Die in Tab. 4 abgebildete Regressionsanalyse zeigt als signifikanten Prädiktor der Ablehnung des selbstgesteuerten Schriftspracherwerbs wie schon 2006 die Unsicher- 
Tab. 2 Stichprobendaten

\begin{tabular}{lllll}
\hline & Lebensalter & \multicolumn{3}{l}{ Berufserfahrung } \\
& 2006 & 2016 & 2006 & 2016 \\
\hline$N$ & 190 & 124 & 187 & 124 \\
Minimum & 20 & 20 & 1 & 1 \\
Maximum & 58 & 63 & 42 & 43 \\
MW (SD) & $36,1(10,2)$ & $43,70(12,27)$ & $13,2(9,0)$ & $20,28(12,28)$ \\
\hline
\end{tabular}

Tab. 3 Ablehnung des selbstgesteuerten Schriftspracherwerbs (Messwiederholung ausschließlich bei den Teilnehmenden, von denen Daten aller drei MZPs vorliegen)

\begin{tabular}{llllll}
\hline & MZP 1 & MZP 2 & MZP 3 & $F(2 ; 14)$ & Part. $\eta^{2}$ \\
& $M(S D)$ & $M(S D)$ & $M(S D)$ & & \\
\hline Skala & & & & & \\
$\begin{array}{l}\text { Ablehnung des selbstgesteuerten } \\
\text { Schriftspracherwerbs }\end{array}$ & 1,96 & 1,89 & 2,28 & $3,61^{*}$ & 0,21 \\
\hline
\end{tabular}

${ }^{*} p<0,05$; part. $\eta^{2}>0,13$ großer Effekt

Tab. 4 Beta-Gewichte der verschiedenen Einflussvariablen

\begin{tabular}{lll}
\hline & $\begin{array}{l}\text { Abhängige Variable: Einstel- } \\
\text { lung zum selbstgesteuerten } \\
\text { Schriftspracherwerb }(n=105)\end{array}$ & $\begin{array}{l}\text { Abhängige Variable: Ablehnen- } \\
\text { der Umgang mit einem Kind, } \\
\text { das im Kindergarten phonetisch } \\
\text { schreibt }(n=102)\end{array}$ \\
\hline Konstante & $2,30^{* *}$ & $1,56^{*}$ \\
Jahre der Berufstätigkeit & 0,01 & 0,01 \\
Zusammenarbeit mit der Schule & $-0,02$ & $-0,12$ \\
Unsicherheit gegenüber der Schule & $0,35^{*}$ & $0,30^{*}$ \\
Unsicherheit bezüglich Sprach- & $-0,01$ & 0,16 \\
und Schriftsprachentwicklung von & & \\
Kindern & 0,07 & $0,21^{* *}$ \\
Instruierendes Lernkonzept & $-0,32^{*}$ & $-0,33^{*}$ \\
Konstruktivistisches Lernkonzept & $0,15^{* *}$ & $0,27^{* *}$ \\
Korrigiertes R & & $\mathrm{F}(6,95)=7,07$ \\
F-Wert & $\mathrm{F}(6,98)=4,12$ &
\end{tabular}

Nicht standardisierte Koeffizienten ${ }^{*} p<0,05 ;{ }^{* *} p<0,01 ;{ }^{+} p<0,10$

$R^{2}$ erklärte Stichprobenvarianz

heit gegenüber der Schule. Wenn die pädagogischen Fachkräfte unsicher sind, wie kognitive Aktivitäten im Kindergarten von der Schule wahrgenommen werden, lehnen sie phonetisches Schreiben im Kindergarten häufiger ab. Ein konstruktivistisches Lernkonzept hingegen wirkt positiv, denn konstruktivistisch eingestellte Fachkräfte lehnen den selbstgesteuerten Schriftspracherwerb seltener ab. Noch deutlicher werden die Einflüsse bezogen auf das Fallbeispiel eines Kindes, das im Kindergarten phonetisch schreibt. Hier zeigt sich bei $27 \%$ Varianzaufklärung, dass ein konstruktivistisches Lernkonzept dazu beiträgt, dass die pädagogische Fachkraft das Kind unterstützt. Ein instruierendes Lernkonzept und Unsicherheit gegenüber der Schule hingegen hängen mit einer ablehnenden Haltung gegenüber der Tätigkeit zusammen. 
Tab. 5 Kodierungen der offenen Fragen

\begin{tabular}{|c|c|c|}
\hline Code & Ankerbeispiel & $\begin{array}{l}\text { Zahl der } \\
\text { Nennungen }\end{array}$ \\
\hline $\begin{array}{l}\text { Bedeutsamkeit der pho- } \\
\text { nologischen Bewusstheit }\end{array}$ & $\begin{array}{l}\text { „Die phonologische Bewusstheit ist eine wichtige Vorausset- } \\
\text { zung zum Lesen- und Schreibenlernen." }\end{array}$ & 24 \\
\hline $\begin{array}{l}\text { Spielerisches, mitgängi- } \\
\text { ges Lernen }\end{array}$ & „Das ,Lernen' geschieht bei uns im Spiel.“ & 11 \\
\hline $\begin{array}{l}\text { Lesen und Schreiben ist } \\
\text { Sache der Schule }\end{array}$ & „Lesen und Schreiben lernen soll Sache der GS bleiben!“ & 8 \\
\hline $\begin{array}{l}\text { Motivation und Interes- } \\
\text { se an Schriftsprache }\end{array}$ & $\begin{array}{l}\text { „Die ,Vorschulkinder' sind sehr daran interessiert schreiben } \\
\text { zu lernen." }\end{array}$ & 7 \\
\hline Selbsttätigkeit & $\begin{array}{l}\text { „Kinder sollen aktiv tun und arbeiten lernen, durch } \\
\text { be,greifen'!!" }\end{array}$ & 6 \\
\hline $\begin{array}{l}\text { Ganzheitliche Förde- } \\
\text { rung }\end{array}$ & $\begin{array}{l}\text { „Im KiGa werden viele Bereiche gefördert, die Kinder sollen } \\
\text { ganzheitlich gefördert werden.“ }\end{array}$ & 6 \\
\hline $\begin{array}{l}\text { Kritik an vorgegebenen } \\
\text { Programmen }\end{array}$ & „Weg von Sprachprogrammen u. Förderprogrammen“ & 5 \\
\hline $\begin{array}{l}\text { Gute Zusammenarbeit } \\
\text { mit der Grundschule }\end{array}$ & „Zusammenarbeit mit GS ist intensiver und offener.“ & 5 \\
\hline $\begin{array}{l}\text { Kein Wissen über die } \\
\text { spätere Entwicklung } \\
\text { der Kinder in der Schule }\end{array}$ & $\begin{array}{l}\text { „Wie es den Kindern nach dem Kiga in der Schule geht, } \\
\text { bekommen wir nicht mit." }\end{array}$ & 4 \\
\hline $\begin{array}{l}\text { Lesen und Schreiben in } \\
\text { der Kita ist gut }\end{array}$ & $\begin{array}{l}\text { „Deshalb unterstützen wir auch die Kinder voll in ihren In- } \\
\text { teressen \& bieten Material zur phonologischen Bewusstheit } \\
\text { \& dem Schreiben- \& Lesenlernen ganz natürlich in unserem } \\
\text { Alltag an." }\end{array}$ & 4 \\
\hline $\begin{array}{l}\text { Zusammenarbeit mit der } \\
\text { Schule sollte besser sein }\end{array}$ & $\begin{array}{l}\text { „Lediglich die Zusammenarbeit mit der Schule könnte inten- } \\
\text { siver sein." }\end{array}$ & 4 \\
\hline $\begin{array}{l}\text { Ablehnung phoneti- } \\
\text { sches Schreiben }\end{array}$ & $\begin{array}{l}\text { „Ich finde es nicht gut, wenn die Kinder in der Schule } \\
\text { schreiben dürfen, wie sie die Wörter sprechen. Sie sollen } \\
\text { von Anfang an die Rechtschreibung lernen!“" }\end{array}$ & 3 \\
\hline
\end{tabular}

Die Auswertung der offenen Frage des Fragebogens liefert hier mögliche Erklärungen. In Tab. 5 sieht man die Übersicht der häufigsten Kodierungen in absteigender Reihenfolge der Nennungen.

In den offenen Antworten bestätigen sich sowohl die Ergebnisse der eingangs geschilderten Forschungsbefunde als auch der quantitativen Studie. Die Fachkräfte setzen v. a. auf spielerisches Lernen, auf Selbsttätigkeit und Ganzheitlichkeit. Die phonologische Bewusstheit ist ein wichtiger Aspekt, deren Bedeutung in der Wahrnehmung der Fachkräfte sogar gestiegen ist. Programme wie „Hören, Lauschen, Lernen“ (Küspert und Schneider 1999) sind im Kindergarten etabliert und werden größtenteils positiv gesehen.

Fertigkeiten, die sich den schulischen Anforderungen nähern, also v. a. konkretes Lesen und Schreiben, werden eher abgelehnt. Als typische Aussage kann dieses Zitat gelten, in dem die Grenzziehung deutlich wird: „Wenn im Kindergarten Reimen usw. spielerisch geübt wird, merke ich, dass sich die Kinder dann in der Schule ein wenig leichter tun. Aber das Lesen- und Schreibenlernen gehört in die Schule, wir üben zwar mit den Kindern ein wenig Zahlen und Buchstaben, aber das ist Aufgabe der Schule (UDONA)“. Die einzigen zustimmenden Stimmen zu frühem 
Schriftspracherwerb kamen von vier pädagogischen Fachkräften, die alle vier zur Begründung die Montessori-Pädagogik herangezogen haben, wie etwa diese Fachkraft: „Durch das Montessori-Diplom habe ich einen anderen Einblick erworben. Durch das Arbeiten mit dem Sprach-Material lernen Kinder (spielerisch) das Lesen und Schreiben bereits im Kiga schon, und wollen immer mehr lernen (RI19IA)“. Außer diesen vier Personen finden sich in der qualitativen Stichprobe ausschließlich ablehnende Stimmen zu vorschulischem Schriftspracherwerb.

Die Zusammenarbeit mit der Grundschule wird hierbei mehr positiv als negativ gesehen, wenn auch immer noch das Problem besteht, dass die Fachkräfte nicht wissen, wie sich ihre Kinder nach dem Kindergarten entwickeln.

Nennungen zur Förderung von Kindern mit Schwierigkeiten finden sich kaum. Wenn Schwierigkeiten angesprochen werden, dann oft ohne Konsequenz für die eigene Arbeit, wie z. B. hier: „Im Laufe der letzten Jahre konnte ich feststellen, dass Kinder immer öfter, schlampig ' sprechen, weil die Konversation zu Hause oft zu kurz kommt, z. B. Zeitmangel, Migrationshintergrund, falsche Vorbilder (ND07SA)““. Förderung wird am ehesten im Sinne alltagsintegrierter Unterstützung umgesetzt. „Da es immer mehr Kinder gibt, die Auffälligkeiten im Spracherwerb zeigen, legt man verstärkt Wert darauf, mit den Kindern Fingerspiele, Reime, Flüsterspiele, SilbenKlatsch-Spiele in den Alltag einzubauen (UB31UD)“. Die alltagsintegrierte Förderung wird auch als Gegenentwurf zu vorgegebenen Förderprogrammen verstanden, um „Lernen in alltäglichen Situationen zu nützen, keine Programme (AN18TE)“.

Hier zeigt sich mitunter auch eine Ablehnung des phonetischen Schreibens, die weitgehend über das phonetische Schreiben in der Schule begründet wird. So schreibt eine Fachkraft: ,Nicht durch meine Arbeit, aber durch meine ältere Tochter! Sie ist jetzt in der 4. Klasse u. hat quasi die ganze 1. Klasse mit Hilfe dieser Auslauttabelle [sic] schreiben dürfen wie sie wollte! Das zieht sich leider bis heute durch! Uns (auch als Erzieherinnen) wurde ja jahrelang erzählt, wie toll das ist u. ich fand es auch toll, bis meine Tochter eben in die Schule kam! Jetzt ist man ja Gott sei Dank davon wieder abgekommen, meine jüngere Tochter (2. Klasse) hat ,Lernwörter“, die sie zig mal schreibt, die tut sich viel leichter! (OS10NA)“.

Unter Berücksichtigung der vorliegenden Daten kann zusammenfassend Folgendes festgestellt werden: Die pädagogischen Orientierungen zum Schriftspracherwerb im Kindergarten beinhalten eine Sicht ganzheitlicher spielerischer Unterstützung der Kinder. Die phonologische Bewusstheit wird als wichtig erachtet. Bei Vorhandensein eines konstruktivistischen Lernkonzepts und Sicherheit gegenüber der Schule ist auch weniger Ablehnung gegenüber dem vom Kind selbst gesteuerten Schriftspracherwerb bereits im Kindergarten zu erkennen. Eine solche Haltung findet man z. B. bei Montessori-Pädagoginnen. Insgesamt wird selbstgesteuerter Schriftspracherwerb aber eher abgelehnt und zwar signifikant stärker als noch vor zehn Jahren.

\section{Diskussion}

Seit vielen Jahren betont die Forschung die Bedeutung der Vorläuferfähigkeiten. Im Bereich des Schriftspracherwerbs sind es neben den Kompetenzen der phonologischen Informationsverarbeitung v. a. die frühe Schriftkenntnis und das Wissen 
über Schrift. Bei qualitativ hochwertigen Angeboten in diesem Bereich kann der Kindergarten sozial ausgleichend wirken.

In den letzten zehn Jahren hat sich die Einstellung zum Kindergarten als Lernort erhalten. Die Bedeutung der phonologischen Bewusstheit sowie der Förderung dieser Kompetenz, auch mit Programmen, ist bei den Fachkräften im Kindergarten größtenteils anerkannt.

Allerdings ist die erstaunliche Diskrepanz festzustellen, dass die Fachkräfte zwar sehr stark die Bedeutung der Selbsttätigkeit und des Interesses betonen, aber größtenteils stoppen, sobald es um den von Kindern selbst gesteuerten Schriftspracherwerb geht. Hier zeigen die Regressionsanalysen, dass dies auch mit der Unsicherheit gegenüber der Schule sowie einem instruierenden bzw. konstruktivistischem Lernkonzept zusammenhängt. Deutlich zeigt sich dieser Zusammenhang bei den offenen Antworten: Die Zustimmung zum Lesen und Schreiben im Kindergarten kommt ausschließlich von Personen aus dem Montessori-Kontext, wo eine konstruktivistische Lerneinstellung vorherrscht. Die deutlich häufigere Äußerung, dass Lesen und Schreiben Sache der Schule sei, ist hingegen wie schon vor zehn Jahren verknüpft mit einer Unsicherheit gegenüber der Schule.

Als einzige signifikante Veränderung findet sich in dieser Studie nach zehn Jahren die zunehmende Ablehnung des selbstgesteuerten Schriftspracherwerbs. Die Studie bestätigt also die eingangs dargestellten Befunde. Die Vorläuferfähigkeiten sind den pädagogischen Fachkräften wichtig, doch je näher die Kompetenzen der Kinder in Richtung Lesen und Schreiben rücken, desto eher werden sie abgelehnt. Es ist hier nicht nur ein „,normatives Konzept von Kindheit“, wie Plehn (2013, S. 228) es nennt, sondern auch vom Kindergarten als ganzheitlichem Gegenentwurf zur Schule zu sehen. Das ist verständlich und doch verkennt diese Sichtweise die hohe Bedeutung und Chance der vorschulischen Kompetenzentwicklung.

In den offenen Antworten wird phonetisches Schreiben mit der Schule, auch mit Erfahrungen bei den eigenen Kindern verbunden, was einen Hinweis auf die Quelle dieser Skepsis geben kann. Dabei ist selbstgesteuertes Lesen und Schreiben vor Schulbeginn nicht schädlich, sondern für die Kinder extrem motivierend und lernwirksam. Aber natürlich muss die Schule mit Angeboten zum Orthografieerwerb darauf aufbauen.

Dass die kompensatorische und förderliche Wirkung des Kindergartens in den offenen Aussagen kaum angesprochen wird, trotz der Veränderungen der letzten zehn Jahre (wie etwa Fluchtmigration), kann man zumindest konstatieren und zum Gegenstand von Fortbildung machen.

Insgesamt müsste vermutlich in der Aus- und Weiterbildung an den subjektiven Theorien direkt gearbeitet werden, um bei einzelnen Fachkräften die Einstellung zum vorschulischen Schriftspracherwerb und zur förderlichen Wirkung früher Bildung (auch von Programmen) zu verändern.

Funding Open Access funding provided by Projekt DEAL. 
Open Access Dieser Artikel wird unter der Creative Commons Namensnennung 4.0 International Lizenz veröffentlicht, welche die Nutzung, Vervielfältigung, Bearbeitung, Verbreitung und Wiedergabe in jeglichem Medium und Format erlaubt, sofern Sie den/die ursprünglichen Autor(en) und die Quelle ordnungsgemäß nennen, einen Link zur Creative Commons Lizenz beifügen und angeben, ob Änderungen vorgenommen wurden.

Die in diesem Artikel enthaltenen Bilder und sonstiges Drittmaterial unterliegen ebenfalls der genannten Creative Commons Lizenz, sofern sich aus der Abbildungslegende nichts anderes ergibt. Sofern das betreffende Material nicht unter der genannten Creative Commons Lizenz steht und die betreffende Handlung nicht nach gesetzlichen Vorschriften erlaubt ist, ist für die oben aufgeführten Weiterverwendungen des Materials die Einwilligung des jeweiligen Rechteinhabers einzuholen.

Weitere Details zur Lizenz entnehmen Sie bitte der Lizenzinformation auf http://creativecommons.org/ licenses/by/4.0/deed.de.

\section{Literatur}

Abry, T., Latham, S., Bassok, D., \& LoCasale-Crouch, J. (2015). Preschool and kindergarten teachers' beliefs about early school competencies: misalignment matters for kindergarten adjustment. Early Childhood Research Quarterly, 31, 78-88.

Bayerisches Staatsministerium für Arbeit und Sozialordnung, Familie und Frauen (Hrsg.). (2006). Der bayerische Erziehungs- und Bildungsplan für Kinder in Tageseinrichtungen bis zur Einschulung. Weinheim, Basel: Beltz.

Becker, B. (2010). Wer profitiert mehr vom Kindergarten? Die Wirkung der Kindergartenbesuchsdauer und Ausstattungsqualität auf die Entwicklung des deutschen Wortschatzes bei deutschen und türkischen Kindern. Kölner Zeitschrift für Soziologie und Sozialpsychologie, 62, 139-163.

Becker, R. (2016). Bildungseffekte vorschulischer Erziehung und Elementarbildung: Bessere Bildungschancen für Arbeiter- und Migrantenkinder? In R. Becker \& W. Lauterbach (Hrsg.), Bildung als Privileg: Erklärungen und Befunde zu den Ursachen der Bildungsungleichheit (5. Aufl. S. 145-181). Wiesbaden: VS.

Becker-Stoll, F., \& Wertfein, M. (2013). Qualitätsmessung und Qualitätsentwicklung in Kindertageseinrichtungen. In M. Stamm \& D. Edelmann (Hrsg.), Handbuch frühkindliche Bildungsforschung (S. 845-856). Wiesbaden: Springer.

Brandl, T. (2016). Kindliches Lernen und pädagogisches Handeln im Kindergarten. Subjektive Theorien angehender Kindheitspädagoginnen und Kindheitspädagogen. Berlin: Logos.

Brühwiler, C., \& Helmke, A. (2018). Determinanten der Schulleistung. In D. H. Rost, J. R. Sparfeldt \& S. R. Buch (Hrsg.), Handwörterbuch Pädagogische Psychologie (5. Aufl. S. 78-92). Weinheim: Beltz.

v. Bülow, K. (2011). Anschlussfähigkeit von Kindergarten und Grundschule. Rekonstruktion von subjektiven Bildungstheorien von Erzieherinnen und Lehrerinnen. Bad Heilbrunn: Klinkhardt.

Diamond, K. E., Gerde, H. K., \& Powell, D. R. (2008). Development in early literacy skills during the prekindergarten year in Head Start: Relations between growth in children's writing and understanding of letters. Early Childhood Research Quarterly, 23, 467-478.

Ehlich, K., Bredel, U., \& Reich, H.H. (2008). Referenzrahmen zur altersspezifischen Sprachaneignung. Bildungsforschung, Bd. 29/I. Bonn und Berlin: BMBF.

Ehri, L., \& Roberts, T. (2006). The roots of learning to read and write: acquisition of letters and phonemic awareness. In S. B. Neuman \& D. K. Dickinson (Hrsg.), Handbook of early literacy research (Bd. 2, S. 113-134). New York: Guilford.

Ennemoser, M., Marx, P., Weber, J., \& Schneider, W. (2012). Spezifische Vorläuferfertigkeiten der Lesegeschwindigkeit, des Leseverständnisses und des Rechtschreibens. Evidenz aus zwei Längsschnittstudien vom Kindergarten bis zur 4. Klasse. Zeitschrift für Entwicklungspsychologie und Pädagogische Psychologie, 44, 53-67.

Faust, G. (Hrsg.). (2013). Einschulung. Ergebnisse aus der Studie „Bildungsprozesse, Kompetenzentwicklung und Selektionsentscheidungen im Vorschul- und Schulalter (BiKS)“. Münster: Waxmann.

Fina, I. (2017). Einschulung mit fünf Jahren? Eine Längsschnittstudie zur Entwicklung vorvorzeitig eingeschulter Kinder in der Grundschule. Bad Heilbrunn: Klinkhardt.

Fuchs-Rechlin, K., \& Bergmann, C. (2014). Der Abbau von Bildungsbenachteiligung durch Kindertagesbetreuung für unter 3-Jährige - zwischen Wunsch und Wirklichkeit. Zeitschrift für Erziehungswissenschaft, 17(2), 95-118. 
v. Goldammer, A., Mähler, C., Bockmann, A.-K., \& Hasselhorn, M. (2010). Vorhersage früher Schriftsprachleistungen aus vorschulischen Kompetenzen der Sprache und der phonologischen Informationsverarbeitung. Zeitschrift für Entwicklungspsychologie und pädagogische Psychologie, 42(1), 48-56.

Hamre, B. K., Pianta, R.C., Burchinal, M., Field, S., LoCasale-Crouch, J., Downer, J.T., Howes, C., LaParo, K., \& Scott-Little, C. (2012). A course on effective teacher-child interactions: effects on teacher beliefs, knowledge, and observed practice. American Educational Research Journal, 49(1), 88-123.

Hasselhorn, M., \& Gold, A. (2017). Pädagogische Psychologie (4. Aufl.). Stuttgart: Kohlhammer.

Kotzerke, M., Röhricht, V., Weinert, S., \& Ebert, S. (2013). Sprachlich-kognitive Kompetenzunterschiede bei Schulanfängern und deren Auswirkungen bis Ende der Klassenstufe 2. In G. Faust (Hrsg.), Einschulung. Ergebnisse aus der Studie „Bildungsprozesse, Kompetenzentwicklung und Selektionsentscheidungen im Vorschul- und Schulalter (BiKS) “ (S. 111-135). Münster: Waxmann.

Küspert, P., \& Schneider, W. (1999). Hören, lauschen, lernen. Sprachspiele für Kinder im Vorschulalter. Würzburger Trainingsprogramm zur Vorbereitung auf den Erwerb der Schriftsprache. Göttingen: Vandenhoeck \& Ruprecht.

Lenel, A. (2005). Schriftspracherwerb im Vorschulalter. Eine entwicklungspsychologische Längsschnittstudie. Weinheim, Basel: Beltz.

Liebers, K. (2016). Erwerb von Early Literacy unter dem Fokus Bildungsbenachteiligung. In E. Inckemann \& R. Sigel (Hrsg.), Diagnose und Förderung von bildungsbenachteiligten Kindern im Schriftspracherwerb (S. 21-34). Bad Heilbrunn: Klinkhardt.

Lynch, J., \& Owston, R. (2015). Preschool teachers' beliefs about the teaching and learning of language and literacy: implications for education and practice. International Research in Early Childhood Education, 6(1), 91-111.

Mischo, C., Wahl, S., Hendler, J., \& Strohmer, J. (2012). Pädagogische Orientierungen angehender frühpädagogischer Fachkräfte an Fachschulen und Hochschulen. Frühe Bildung, 1, 34-44.

Neumann, M., Becker, M., \& Maaz, K. (2014). Soziale Ungleichheiten in der Kompetenzentwicklung in der Grundschule und der Sekundarstufe I. Zeitschrift für Erziehungswissenschaft, 17, 167-203.

Plehn, M. (2013). Frühzeitig, fristgemäß oder verzögert? Pädagogische Orientierungen von Erzieherinnen bei der Einschulung. In G. Faust (Hrsg.), Einschulung. Ergebnisse aus der Studie „Bildungsprozesse, Kompetenzentwicklung und Selektionsentscheidungen im Vorschul- und Schulalter (BiKS) “ (S. 221-236). Münster: Waxmann.

Pohlmann-Rother, S., Kratzmann, J., \& Faust, G. (2013). Schulfähigkeit in der Sicht von Eltern, Erzieherinnen und Lehrkräften. In G. Faust (Hrsg.), Einschulung. Ergebnisse aus der Studie „Bildungsprozesse, Kompetenzentwicklung und Selektionsentscheidungen im Vorschul- und Schulalter (BiKS)“ (S. 153-169). Münster: Waxmann.

Rank, A. (2008). Subjektive Theorien von Erzieherinnen zu vorschulischem Lernen und Schriftspracherwerb. Berlin: Wissenschaftlicher Verlag Berlin.

Rank, A. (2009). Subjektive Theorien von Erzieherinnen zu vorschulischem Lernen und Schriftspracherwerb. Zeitschrift für Grundschulforschung, 2(1), 146-159.

Rauschenbach, T. (2009). Zukunftschance Bildung. Familie, Jugendhilfe und Schule in neuer Allianz. Weinheim: Beltz.

Roßbach, H.-G. (2005). Effekte qualitativ guter Betreuung, Bildung und Erziehung im frühen Kindesalter auf Kinder und ihre Familien. In Sachverständigenkommission Zwölfter Kinder- und Jugendbericht (Hrsg.), Bildung, Betreuung und Erziehung von Kindern unter sechs Jahren (Bd. 1, S. 55-17). München: DJI.

Schneider, W. (2004). Frühe Entwicklung von Lesekompetenz: Zur Relevanz vorschulischer Sprachkompetenzen. In U. Schiefele, C. Artelt, W. Schneider \& P. Stanat (Hrsg.), Struktur, Entwicklung und Förderung von Lesekompetenz. Vertiefende Analysen im Rahmen von PISA 2000 (S. 13-36). Wiesbaden: VS.

Stern, E., \& Grabner, R. (2014). Die Erforschung menschlicher Intelligenz. In L. Ahnert (Hrsg.), Theorien in der Entwicklungspsychologie (S. 174-201). Heidelberg: Springer.

Tietze, W. (Hrsg.). (1998). Wie gut sind unsere Kindergärten? Neuwied, Berlin: Luchterhand.

Wang, J., Elicker, J., McMullen, M., \& Shuyang, M. (2008). Chinese and American preschool teachers' beliefs about early childhood curriculum. Early Child Development and Care, 178(3), 227-249.

Wehner, F., \& Kratzmann, J. (2013). Einstellungen von Eltern und Erzieherinnen zur Förderung von Kindern im Alter von drei bis sechs Jahren. In G. Faust (Hrsg.), Einschulung. Ergebnisse aus der Studie „Bildungsprozesse, Kompetenzentwicklung und Selektionsentscheidungen im Vorschul- und Schulalter (BiKS)“ (S. 83-96). Münster: Waxmann. 
Weinert, F. E. \& Stefanek, J. (1997). Entwicklung vor, während und nach der Grundschulzeit. Ergebnisse aus dem SCHOLASTIK-Projekt. In: Weinert, F.E. \& Helmke, A. (Hrsg.), Entwicklung im Grundschulalter (S. 423-451). Weinheim: Beltz.

Wen, X. I., Elicker, J.G., \& McMullen, M. (2011). Early childhood teachers' curriculum beliefs: are they consistent with observed classroom practices? Early Education \& Development, 22(6), 945-969. 